\title{
Evaluation of an intensive education program on the treatment of tobacco-use disorder for pharmacists: a study protocol for a randomized controlled trial
}

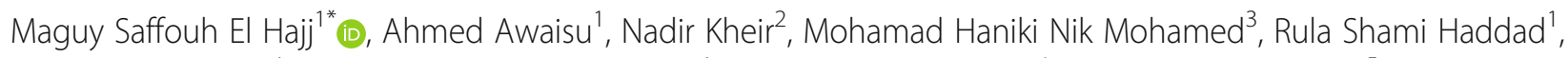
Rana Ahmed Saleh', Noora Mohammed Alhamad', Ahmad Mohd Almulla ${ }^{4}$ and Ziyad R. Mahfoud ${ }^{5}$

\begin{abstract}
Background: Tobacco use is presently responsible for the death of over seven million people across the world. In Qatar, it is one of the main causes of premature deaths and preventable diseases. To reduce tobacco use, Qatar has ratified the World Health Organization (WHO)'s Framework Convention on Tobacco Control (FCTC) and has implemented many tobacco-control initiatives. In spite of these measures, tobacco use is still considered a public health threat in Qatar. Pharmacists practicing in retail/community pharmacy settings are often the first port of call for individuals requiring general health advice. Evidence has proven that they have a pivotal role in health promotion and disease prevention including tobacco cessation. However, pharmacists in Qatar are not actively involved in tobacco control and many have not received any education or training about smoking cessation counseling in the past. In an effort to build the capacity of pharmacists towards tobacco control in Qatar, the aim of the proposed study is to design, implement, and evaluate an intensive education program on tobacco dependence treatment for pharmacists in Qatar.
\end{abstract}

Methods/design: The study will be a prospective randomized controlled trial comparing an intensive tobacco-related education program versus non-tobacco-related training on pharmacists' tobacco-use-related knowledge, attitudes, selfefficacy, and skills. Community pharmacists practicing in Qatar will be eligible for participation in the study. A random sample of pharmacists will be selected for participation. Consenting participants will be randomly allocated to intervention or control groups. Participants in the intervention group will receive an intensive education program delivered by a multi-disciplinary group of educators, researchers, and clinicians with expertise in tobacco cessation. A short didactic session on a non-tobacco-related topic will be delivered to pharmacists in the control group. The study has two primary outcomes: post-intervention tobacco-related knowledge and post-intervention skills for tobacco cessation assessed using a multiple-choice-based evaluation instrument and an Objective Structured Clinical Examination (OSCE), respectively. The secondary study outcomes are post-intervention attitudes towards tobacco cessation and selfefficacy in tobacco-cessation interventions assessed using a survey instrument. An additional secondary study outcome is the post-intervention performance difference in relation to tobacco-cessation skills in the practice setting assessed using the simulated client approach.

Discussion: If demonstrated to be effective, this education program will be considered as a model that Qatar and the Middle East region can apply to overcome the burden of tobacco-use disorder.

Trial registration: ClinicalTrials.gov, ID: NCT03518476. Registered on 8 May 2018. Version 1/22 June 2018.

Keywords: Qatar, Education program, Tobacco control, Smoking cessation, Pharmacist

\footnotetext{
* Correspondence: maguyh@qu.edu.qa

${ }^{1}$ College of Pharmacy, Qatar University, Doha 2713, Qatar

Full list of author information is available at the end of the article
}

(c) The Author(s). 2019 Open Access This article is distributed under the terms of the Creative Commons Attribution 4.0 International License (http://creativecommons.org/licenses/by/4.0/), which permits unrestricted use, distribution, and reproduction in any medium, provided you give appropriate credit to the original author(s) and the source, provide a link to the Creative Commons license, and indicate if changes were made. The Creative Commons Public Domain Dedication waiver (http://creativecommons.org/publicdomain/zero/1.0/) applies to the data made available in this article, unless otherwise stated. 


\section{Background}

Tobacco use is one of the leading causes of premature morbidity and mortality in the world causing over seven million deaths a year [1]. With the current trend, it is projected that tobacco use will cause more than eight million deaths per year by 2030 [2]. Tobacco-use cessation entails many short- and long-term health benefits for the user, the healthcare system, and to society [3]. These benefits include, but are not limited to, reduced risk of lung cancer and other types of cancers, cardiovascular diseases, and lung diseases such as chronic obstructive pulmonary disease [4]. Despite this, tobacco-use cessation is challenging and difficult to achieve without the support of a healthcare professional. Tobacco-cessation interventions offered by healthcare professionals are more effective in comparison with self-help [5]. Pharmacists practicing in community pharmacies are among the most accessible healthcare professionals by the public for providing advice on health and self-care. Evidence has proven that pharmacists have a pivotal role in health promotion and disease prevention including tobacco-cessation interventions [6, 7]. Indeed with the emergence of the pharmaceutical care concept more than two decades ago, the focus of pharmacists has shifted from product-oriented practice to patient-centered care [8]. Many pharmacists, worldwide, have started applying behavioral interventions to improve health outcomes [6]. In addition, prescription and over-the-counter pharmacotherapies for smoking cessation are readily available in community and retail pharmacies. Nicotine replacement therapy (NRT) is an example of an over-thecounter smoking cessation aid available in most countries including Qatar. Prescription smoking cessation medications include bupropion and varenicline. The availability of these medications provides pharmacists with an opportunity to help patients quit smoking and to combat tobacco use in the community [9]. Pharmacist-delivered tobacco-cessation interventions have been described in the literature [9-15]. For example, Saba et al. have conducted a meta-analysis of the effectiveness of pharmacistdelivered smoking cessation interventions within community pharmacy. The analysis has demonstrated that community pharmacy-based smoking cessation interventions can improve smoking abstinence rates [9].

The International Pharmaceutical Federation (FIP) issued a policy statement in relation to the pharmacist's role in reducing tobacco use in the community [16]. The U.S. Department of Health and Human Service clinical practice guidelines for treating tobacco use and dependence include strategies to assist clinicians in delivering tobacco-cessation interventions [5]. Furthermore, healthcare providers, including pharmacists, were highly endorsed for delivering tobacco-cessation services in the American Society of Health-System Pharmacists' (ASHP) Therapeutic Position Statement on the Cessation of Tobacco Use [17].
Tobacco use is a major preventable cause of mortality and morbidity in Qatar. As per the 2013 Global Adult Tobacco Survey (GATS), $12.1 \%$ of adults and $20.2 \%$ of men smoke tobacco, and $55.4 \%$ of smokers smoke an average of 16 cigarettes per day [18]. Moreover, according to the 2013 Global Youth Tobacco Survey (GYTS), 15.7\% of school students in Qatar within the age range of 13 and 15 years currently use tobacco [19]. Tobacco-induced diseases including cardiovascular diseases (CVDs) are highly prevalent in Qatar. The mortality rate from CVDs from 2011 to 2013 was 8.3 per 100,000 for Qatari males aged 20-44 years [20]. Furthermore, lung cancer is one of the main causes of cancer-related mortality in males accounting for 17.4\% of cancer-related deaths in 2014 [21]. Qatar residents purchase at least one billion cigarettes annually and 150 million dollars are spent per year to cover the hospital fees for smoking-related diseases [22]. To reduce the use of tobacco, Qatar has ratified the World Health Organization (WHO)'s Framework Convention on Tobacco Control (FCTC) [23] and has implemented several initiatives to reduce tobacco use including banning all forms of tobacco advertisement in media, prohibiting smoking in enclosed public areas, forbidding the sale of cigarettes to children under the age of 18 years, introducing graphic/pictorial health warnings on cigarette packs, and others [23, 24]. Despite these initiatives, tobacco use is considered a public health threat in Qatar. Qatar pharmacists and other healthcare providers have excellent opportunities to reduce tobacco use in the country. Currently, Qatar has over 1000 practicing community pharmacists and smoking cessation aids including NRT and varenicline are available over-the-counter in community pharmacies in Qatar [25]. Despite this, published studies have shown that community pharmacists in Qatar are not actively contributing to tobacco control [26]. Only $21 \%$ of community pharmacists in Qatar always or most of the time ask patients about their tobacco use or smoking status and less than $50 \%$ always or most of the time provide counseling to the purchasers of NRT. Furthermore, $89 \%$ of the community pharmacists reported that they did not receive any kind of education or training about smoking cessation counseling in the past. However, $85 \%$ of the pharmacists were interested in receiving additional training related to smoking cessation competencies/skills including initiating discussions with patients about smoking, assessing patients' nicotine dependence, counseling patients using cognitive behavioral techniques, and other topics [26].

To be competent in providing tobacco-cessation interventions and to offer effective tobacco-cessation programs, pharmacists should have the necessary competence, skills, and confidence in relation to tobacco cessation. Several educational interventions were designed and implemented to enhance the knowledge and 
skills of healthcare professionals in relation to tobacco cessation [27]. Yet the majority of these interventions were implemented and assessed outside the Middle East (ME) region and did not target pharmacists. In addition, these interventions were only delivered by one group of professionals (e.g., nurses, physicians, or pharmacists) with expertise in smoking cessation programs. In an effort to build the capacity of pharmacists on tobacco-use treatment, the aim of this study is to design, implement, and evaluate an intensive education program on tobacco treatment for pharmacists in Qatar. We hypothesize that pharmacists who participate in the program will have better knowledge, attitudes, perceived self-efficacy and skills in relation to tobacco-use treatment than pharmacists who receive no such program (control group).

\section{Study objectives}

The aim of this randomized controlled study is to design, implement, and evaluate an intensive education program provided by team of health professional educators on tobacco-use treatment for pharmacists in Qatar.

The primary objectives of the study are to assess the effectiveness of the program on pharmacists' knowledge and skills in relation to tobacco and tobacco cessation assessed using a multiple-choice-based evaluation instrument and an Objective Structured Clinical Examination (OSCE), respectively. The secondary objectives are to assess the effectiveness of the program on pharmacists' attitudes toward tobacco cessation and perceived selfefficacy (i.e.. confidence) in tobacco-cessation interventions, assessed using a survey instrument, and to assess the effectiveness of the program on pharmacists' tobacco-cessation skills in the practice setting, assessed using the simulated client approach.

\section{Methods/design Study design}

The study is a prospective randomized controlled trial comparing the effectiveness of the education program, versus non-tobacco-related training, on pharmacists' tobacco-related knowledge, attitudes, self-efficacy, and skills. The study methodology and procedures are shown in Fig. 1. The protocol is developed in accordance with the Standard Protocol Items: Recommendations for Interventional Trials guidelines (SPIRIT; Fig. 2 and Additional file 1).

The evaluation of the effectiveness of the program will be conducted using multiple strategies as follows:

- Assessment of pharmacists' knowledge, attitudes, and self-efficacy before and after the training program
- Assessment of pharmacists' skills using an authentic method (i.e., an OSCE) directly after the completion of the training program

- Assessment of pharmacists' skills in the practice setting 3 to 6 months post training program using a simulated client approach

\section{Study-eligible participants}

All community pharmacists practicing in Qatar are eligible for participation in the study. There are no specific exclusion criteria for participants' enrollment in the study.

\section{Recruitment of participants and randomization}

Information about the study and its purpose were made available to the Ministry of Public Health, which subsequently made available a database that has contact details of all community pharmacists practicing in the country (around 1000 pharmacists). A stratified random sample of 200 pharmacists was obtained from the list. Stratification was done by pharmacy so that no more than one pharmacist from each pharmacy would participate. Invitations to participate in the study will be made by the study research assistants (RAs) over the phone or in writing. Those who express interest will be sent a Participant Information Sheet (PIS) and a consent form. Consenting participants will be randomly allocated to intervention or control groups using permuted block randomization with blocks of size 2, 4, and 6 . Randomization will be made by the study statistician who is not involved in the recruitment of the pharmacist and will be concealed for the RAs recruiting the pharmacists. Blinding of study participants in relation to their allocation to the study groups will not be possible given the nature of the intervention.

\section{Intervention group}

Participants in the intervention group will receive an intensive education program delivered by a group of educators, researchers, and clinicians with expertise in tobacco control and tobacco dependence treatment. The program will be delivered at Qatar University over 4 days (run over two weekends) with an average of eight contact hours per day (i.e., approximately 32 contact hours). The program will target the Core Competencies for Evidence-based Treatment of Tobacco Dependence by the Association for the Treatment of Tobacco use and Dependence (ATTUD) [28].

The training curriculum will cover the following topics:

- Tobacco-use epidemiology in Qatar and globally

- Risks and consequences of tobacco use

- Benefits of quitting tobacco use

- Principles of nicotine addiction 


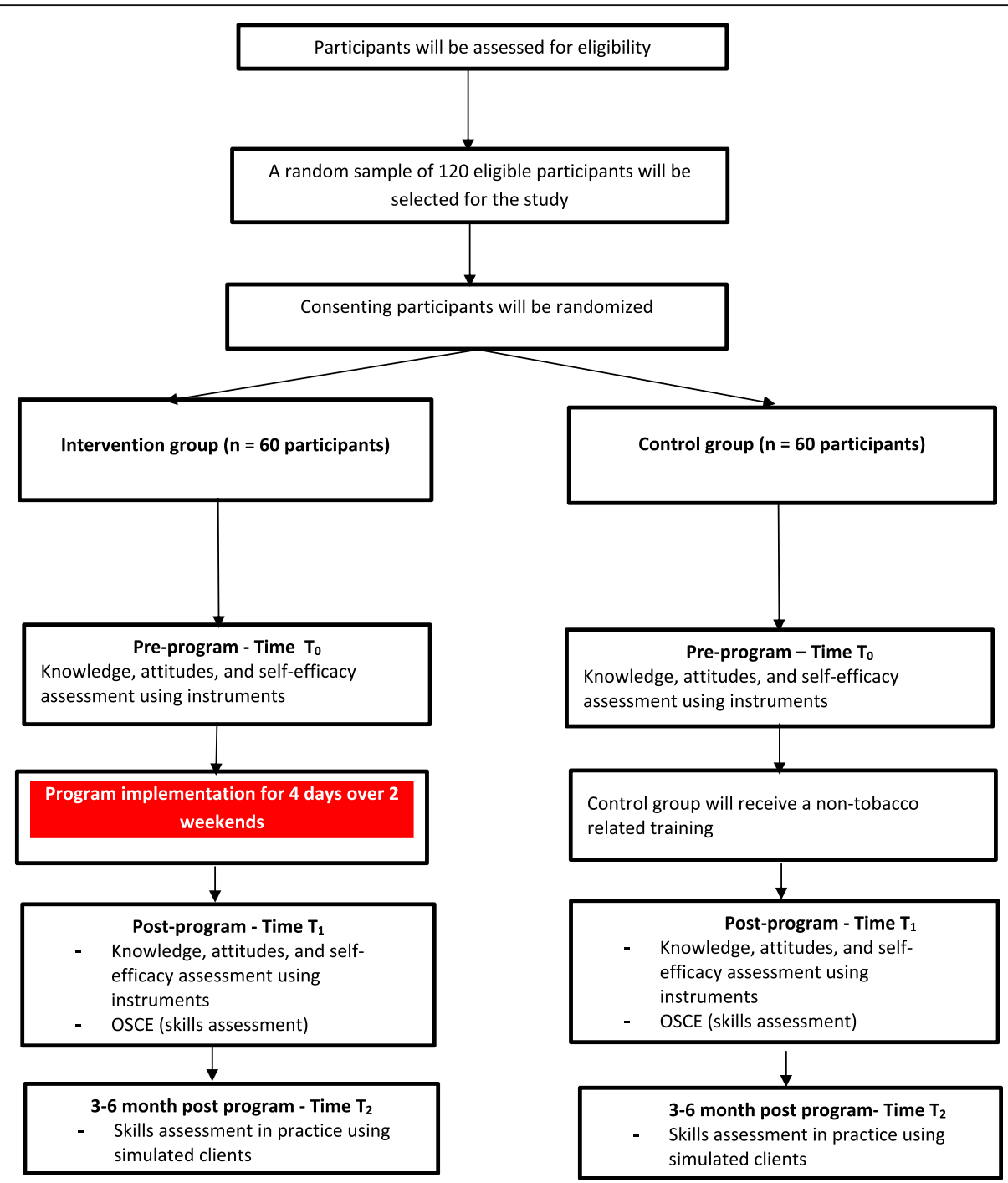

Fig. 1 A flow chart of the study methodology and processes

- Role of pharmacist in implementing tobaccocessation interventions

- Transtheoretical model (TTM) for behavior change as applied to tobacco-use cessation

- Application of the TTM to provide tobacco cessation targeted to the patient's readiness to change

- Behavioral modification techniques for tobacco cessation

- Use of NRT including: indications, the choice of dosage form, dosing regimen, duration of therapy, adverse effects, precautions, contraindications, tapering, pharmacokinetic and pharmacodynamic drug interactions, and patient counseling points

- Pharmacist and patient information on NRT and other cessation aids
- Use of alternative medications (varenicline, bupropion, etc.) and modalities for cessation of tobacco use

- Nicotine withdrawal symptoms

- General verbal and nonverbal communication skills

- Patient counseling and interviewing techniques including the ABC (ask, brief advice, cessation treatment) smoking cessation strategy and the 5A's counseling process (ask, advise, assess, assist, and arrange)

- Development of a personalized action plan to assist the patient with smoking cessation

- Documentation in the patient profile

- Tobacco use and cessation in special populations (e.g., young patients, elderly, pregnant women, and others)

The program will be provided through didactic lectures and through active learning strategies including 


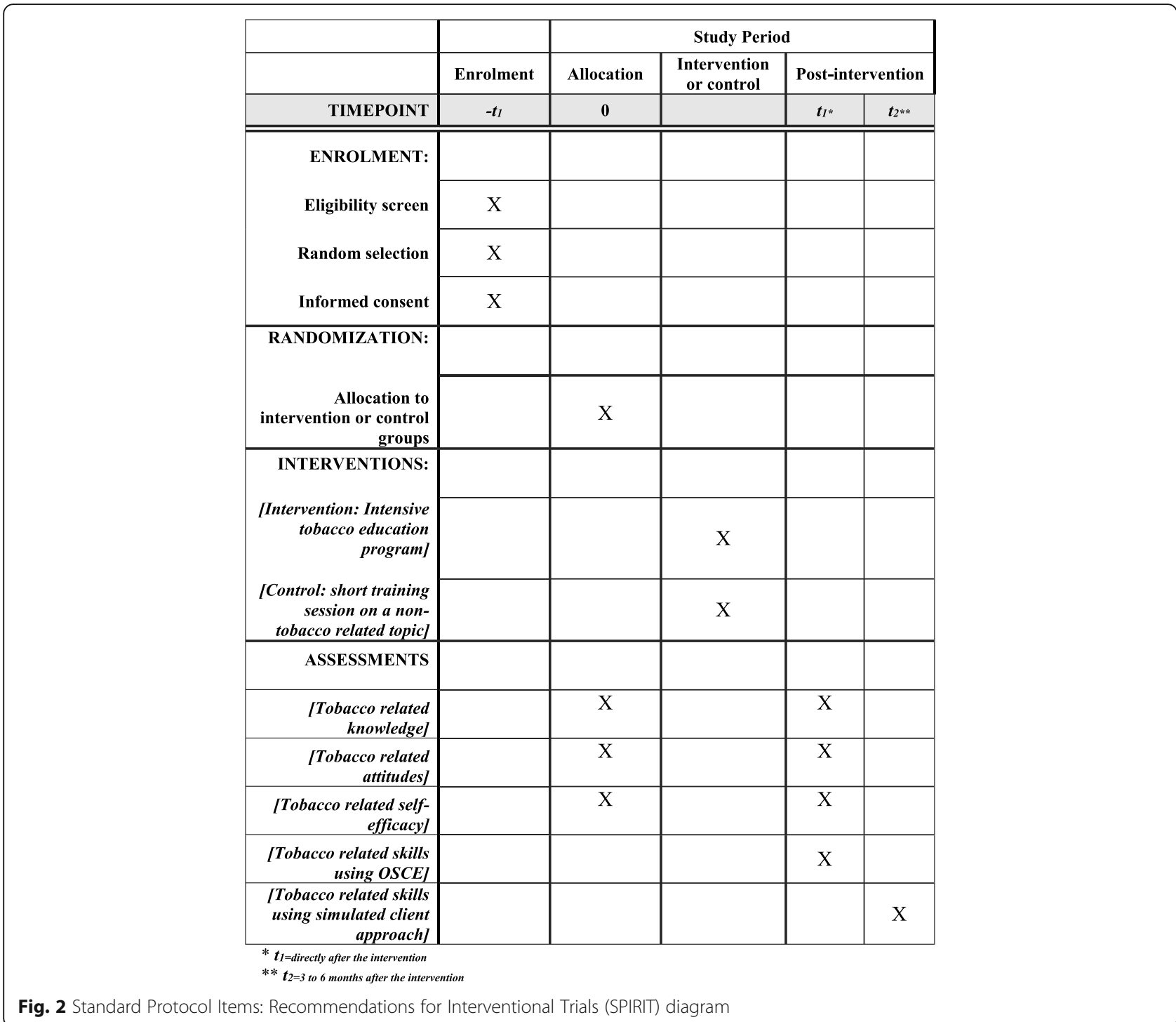

problem-based learning (PBL) exercises using case scenarios, group discussions, games, role plays, videos, simulated practical applications with peers and standardized patients, self and peer debriefing and performance feedback activities. At the end of the program, the pharmacists will be provided with a video recording of all the program content, copies of tools that they can implement in practice and information on online resources containing additional tobaccocessation-related content. They will also be encouraged to contact the research team at any time if they have any questions or concerns. At the conclusion of the training program, all the pharmacists in the intervention group will receive continuous education (CE) units accredited by Qatar Council for Healthcare Practitioners (QCHP) of the Qatar Ministry of Public Health.

\section{Control group}

A short didactic session on a non-tobacco-related topic will be delivered at Qatar University to pharmacists in the control group to avoid contaminating this group with elements that could change their behavior and shift it to another level not representative of their tobacco-cessationrelated "current practice" or "usual care." Pharmacists in the control group will also receive CE units accredited by Qatar Council for Healthcare Practitioners (QCHP) of the Qatar Ministry of Public Health. Examples of potential topics that will be covered in the control group are in relation to women's health such as contraception.

\section{Outcome measures}

The study has two primary outcomes: post-intervention tobacco-related knowledge and post-intervention skills for tobacco cessation assessed using a multiple-choice-based 
evaluation instrument and an OSCE, respectively. The secondary study outcomes are post-intervention attitudes towards tobacco cessation and self-efficacy in tobaccocessation interventions assessed using a survey instrument. An additional secondary study outcome is the post-intervention performance difference in relation to tobacco-cessation skills in the practice setting assessed using the simulated client approach.

\section{Pre- and post-program assessment of pharmacists' knowledge, attitudes, and self-efficacy Study procedures}

This evaluation of the program effectiveness will utilize a before-and-after design between the two groups. The 4-day education program for tobacco treatment will serve as the intervention. Pre-tested instruments will be used to assess knowledge, attitudes and perceived self-efficacy (i.e., confidence) among intervention participants and control participants before and after the program.

\section{Setting}

This evaluation will be conducted in the same venue as the training program (i.e., Qatar University).

\section{Assessment implementation}

The assessment instruments will be distributed to participants in both groups prior to, and following, the program. The assessment will be likely online using a survey software. Participants will be allowed a certain predetermined time to complete the evaluations. Unique participants' codes will be created to link the pre to post data for the same participant.

\section{Post-program assessment of pharmacists' skills using the OSCE \\ Design and structure of the OSCE}

Effective provision of tobacco-cessation intervention requires significant amount of skills in addition to the relevant knowledge. The proposed program has been carefully designed to cover a set of skills and competencies. Assessing participants' practical skills using the traditional assessment methods (e.g., multiple choice questions (MCQs) or short essay) may not be appropriate in assessing skills associated with tobacco cessation An alternative means to assess a pharmacist's practical skills in the delivery of effective tobacco cessation is the use of an authentic, performance-based assessments, such as the OSCE. Examples of skills to be assessed include communication skills in general, counseling skills, professional practice and ethics, and interviewing skills. We propose a five- to eight-station OSCE that will target core competencies and skills covered in the program. Participants in both groups will do the OSCE. In the OSCE, each participant will be allocated $10 \mathrm{~min}$ to interact with a standardized patient (SP) who will be trained using a validated script. Performance of participants will be assessed using validated assessment checklists. The cases and the assessment checklists will be reviewed for content and face validity by at least two faculty members who have expertise in tobacco cessation and suggested changes will be incorporated. Assessors for each station will be trained on using the checklists and will be blinded in relation to the study participants' groups. The OSCE will be conducted in the same venue as the training program.

\section{Three- to six-month post-program assessment of pharmacists' skills using simulated client approach}

In this phase, the study will evaluate whether participants in the intervention and control groups will offer appropriate tobacco-cessation counseling and will recommend proper tobacco-cessation aids in their practice setting. Using a simulated client approach to data collection 3 to 6 months post training program, the study will assess the appropriateness of advice and recommendations given to a simulated client who asks for assistance in quitting smoking. The simulated client method is recognized and used internationally for assessing the quality of services offered by pharmacists including smoking cessation [29-32]. Simulated clients using two designed and validated case scenarios will visit participants in both groups. The clients will be selected to resemble the sociodemographic and practice characteristics of the Qatar population and its residents. Objective assessment forms will be developed using tobacco-cessation guidelines including the U.S. Department of Health and $\mathrm{Hu}$ man Service clinical practice guidelines (CPGs) for treating tobacco use and dependence and the Qatar guidelines for management of tobacco dependence as guides $[5,33]$. Individual items in assessment forms will be completed using dichotomous scales (closed-ended yes/no questions) and will target different aspects of pharmacist performance including gathering information and patient history-taking, assessing the patient, recommending an appropriate treatment plan, and providing counseling. Research assistants will accompany the simulated clients in their visits. They will complete the assessment forms and will record the notes summarizing the encounter. One-to-one training will be provided to simulated clients and RAs by the study research team. Training will offer opportunities for the shoppers to role-play and to become proficient in their assigned scenarios, will help in refining the assessment sheets, in standardizing the process for all participants and in decreasing inter-rater variability. Both simulated clients and RAs will be blinded for the pharmacist group allocation. Each simulated client will enter the community pharmacy and will ask to speak to the pharmacist. The 
client will start with designed queries as per an assigned scenario. The pharmacist will then be given the chance to offer the counseling. After the visit is completed, an explanation of the purpose of the visit and how the collected data will be de-identified will be shared with the pharmacists. Anonymity and confidentiality will be ensured. Consent to participate in this phase of the study will be sought at this time. For pharmacists who decline to participate, information collected during the patient encounter will be discarded.

\section{Sample size estimates}

With 54 pharmacists per group, the study will have an $80 \%$ power to detect an effect size of 0.60 between the study groups for two primary outcomes which are numeric (knowledge and skills scales) using the independent $t$ test with a significance level of $0.025 \%$. In addition, with 54 pharmacists per group, the study will be able to detect a difference of at least $27.5 \%$ between the two study groups for any dichotomous outcome using the chi-square test with $80 \%$ power and a significance level of $5 \%$. Assuming a loss-to-follow-up rate of $10 \%$, the study will randomize 60 pharmacists per group for a total of 120 pharmacists. With over 1000 community pharmacists practicing in Qatar, including 60 pharmacists in each group is achievable. We will select a random sample of 200 pharmacists for recruitment as we anticipate that some pharmacists will not accept to participate. Once the sample size of 120 is reached the recruitment will stop.

\section{Statistical analysis}

Data will be analyzed using IBM SPSS (IBM SPSS ${ }^{\circ}$ for Windows, Version 24.0; IBM Corp, Armonk, NY, USA). The Consolidated Standards of Reporting Trials (CONSORT) guidelines will be followed when analyzing the study data [34]. Demographic and other pharmacist education and practice-related questions, along with baseline knowledge, attitudes, and perceived self-efficacy, will be summarized using means and standard deviations for numeric variables such as age and year of experience and frequency distribution for categorical variables such as gender, country of education, etc. Those will be compared between the study groups so that differences will be used to adjust the primary analyses.

The primary analyses will include comparing postknowledge scores between the two study arms using the independent $t$ test and the skills-related scores on the OSCE using the same method. The secondary analyses will include adjusting the main analyses to any imbalances in baseline variables that are observed between the study arms. This will be done using the linear regression method. Moreover, knowledge scores will be compared within each study group using the paired $t$ test. For outcomes that are dichotomous or that will be dichotomized, such as the percentage of pharmacists with a specific attitude or self-perceived efficacy, then the chisquared test will be used to compare such outcomes between study groups and the McNemar test will be used to compare responses within each study group over time. Performance on the simulated patient will be compared in a similar manner as the outcomes above depending on whether it is dichotomized or not. In addition, repeated-measures analysis (depending on the scale of measurement for each outcome) may be used to detect whether there is an intervention $\times$ time interaction so that difference between study groups is changing over time or not. The level of statistical significance will be set at $5 \%$.

\section{Ethical considerations and data handling}

The study protocol and all related instruments and forms were granted ethical approval by the Qatar University (QU) Institutional Review Board (IRB) (QU-IRB 906-E/18). All data related to the study will be kept confidential and will be retained in a password-protected database maintained along with all related study documentation in a locked cabinet at QU College of Pharmacy. Participation in the study will not cause any harm to participants and, as a result, no data monitoring committee will be assigned. Only the study team will have access to the final study dataset. Any protocol modifications will be communicated with QU IRB. The study results will be presented via poster or oral presentations in international conferences and will be published in peer-reviewed journals. The authors of the final study manuscript will have considerable contributions to the design, implementation, assessment, and reporting of the study.

\section{Monitoring}

The study progress will be monitored closely by the study team. Meetings will be held to guide the study towards its aim and objectives with team involvement. A weekly meeting involving all the team members including RAs will be organized. The study team will be in direct touch with each other and RAs through electronic communication (Google docs and emails). Electronic communication will help in keeping the minutes of the meetings, will provide easy access to any study-related document, and will facilitate online sharing of information. The study team will also conduct ad-hoc meetings at least biweekly to monitor data collection, to check protocol adherence, to review appropriate documentation and to address any issues that might arise. 


\section{Discussion}

Many published studies have demonstrated the impact of tobacco-cessation-related training on pharmacists' knowledge, self-efficacy, confidence, and ability to provide tobacco-cessation counseling [35]. This study will be expected to be the first randomized controlled trial conducted within Qatar and the ME that includes designing, implementing, and evaluating an intensive education program on tobacco-cessation treatment for pharmacists in Qatar. The findings of this study should have far-reaching implications to add to the existing body of knowledge about the management of what is known to be an endemic health hazard costing countries billions of dollars every year and negatively affecting the quality of life of millions of people and their families. In response to the health effects of smoking and its spread, the WHO introduced strategies to combat smoking through the implementation of its Framework Convention on Tobacco Control (FCTC) entitled MPOWER. The six measures under this strategy include monitoring tobacco use and prevention policies; protecting people from tobacco use; offering help to quit tobacco use; warning about the dangers of tobacco; enforcing bans on tobacco advertising, promotion, and sponsorship; and raising taxes on tobacco [1]. Several measures under the "offering help to quit tobacco use" strategy require skilled and trained personnel. The role of pharmacists in helping patients quit smoking is well established. Pharmacists could use their skills and training to become providers of a credible source of health information [9]. However, the skills to help people change their unhealthy behaviors are not generic. The proposed program aims at providing specialist-level training for generalist pharmacists to provide them with the skills and knowledge tailored to assist people who smoke to quit smoking.

Tobacco cessation is one of the most cost-effective of all healthcare interventions and a successful program within the healthcare system will release resources for other needs. The FCTC contains specific obligations concerning tobacco dependence and cessation, including the establishment of programs for diagnosing, counseling, preventing, and treating tobacco dependence [36]. This study builds the foundation for a new form of collaborative interprofessional healthcare team to confront the endemic nature of smoking. The training team consists of expertise from academia and healthcare organizations in the three countries: Qatar, New Zealand, and Malaysia. The training program is anticipated to generate individual healthcare practitioners with the right set of skills and competence to join the current teams at the Tobacco Control Center-WHO Collaborating Center at Hamad Medical Corporation in its efforts within a unique professional approach. This study has the potential to create the right environment for a scheme of continued and seamless smoking cessation care in which referral of patients between primary care and Hamad Medical Corporation (HMC: the main public hospital in Qatar) can be facilitated. The experience and information generated by this study in these aspects should be of interest at the national, regional, and international level.

\section{Trial status}

Preparation for the training program is currently ongoing. It is expected that the recruitment of participants will be held in August 2018 and that the program will be delivered in September 2018.

\section{Additional file}

Additional file 1: Standard Protocol Items: Recommendations for Interventional Trials (SPIRIT) 2013 Checklist: recommended items to address in a clinical trial protocol and related documents*. (DOC $139 \mathrm{~kb}$ )

\section{Abbreviations}

ASHP: American Society of Health-System Pharmacists; ATTUD: Association for the Treatment of Tobacco use and Dependence; CE: Continuous education; CPG: Clinical practice guidelines; FCTC: Framework Convention on Tobacco Control; FIP: International Pharmaceutical Federation; GATS: Global Adult Tobacco Survey; GYTS: Global Youth Tobacco Survey; HMC: Hamad Medical Corporation; MCQ: Multiple choice question; ME: Middle East; NRT: Nicotine replacement therapy; OSCE: Objective Structured Clinical Examination; PBL: Problem-based learning; QCHP: Qatar Council for Healthcare Practitioners; QU: Qatar University; SPIRIT: Standard Protocol Items: Recommendations for Interventional Trials; SPSS: Statistical Package of Social Sciences; TTM: Transtheoretical model; WHO: World Health Organization

\section{Acknowledgements}

Not applicable.

\section{Funding}

This publication was made possible by a collaborative internal grant (QUCGCPH-2018\2019-3) from Qatar University Office of Research and Graduate Studies (email: VPRGS.OFFICE@qu.edu.qa). Its contents are solely the responsibility of the authors and do not necessarily represent the official views of Qatar University.

\section{Availability of data and materials}

The datasets used and/or analyzed during the current study will be available from the corresponding author on reasonable request.

\section{Authors' contributions}

$\mathrm{MH}$ is the principal investigator on the project, leading the design and implementation of the education program. She wrote the project proposal and manuscript. AA is a co-principal investigator and has contributed in the design and implementation of the education program. He has contributed to the conception of the research idea, proposal and education program development, and manuscript preparation. NK has contributed in the design and delivery of the training program, as well as in reviewing and editing the proposal. AM will participate in the training of pharmacists and will assist in following up the progress of the study. $\mathrm{RS}, \mathrm{NH}$, and $\mathrm{RH}$ will help in the preparation for the training program. ZM is the study statistician. He has contributed in the design of the trial and will be guiding the analysis of its data. MHNM will provide technical input, materials for training and assessment as well as participate in the training of pharmacists. All authors read and approved the final manuscript.

\section{Ethics approval and consent to participate}

The study protocol and all associated measurement tools, consent forms, and recruitment procedures were reviewed and approved by Qatar 
University (QU) Institutional Review Board (QU-IRB 906-E/18). The participants will sign written informed consents to participate in this study.

\section{Consent for publication}

Not applicable. The manuscript does not report individual data.

\section{Competing interests}

The authors declare that they have no competing interests.

\section{Publisher's Note}

Springer Nature remains neutral with regard to jurisdictional claims in published maps and institutional affiliations.

\section{Author details}

${ }^{1}$ College of Pharmacy, Qatar University, Doha 2713, Qatar. ${ }^{2}$ School of Pharmacy, Faculty of Medical and Health Sciences, University of Auckland, Auckland, New Zealand. ${ }^{3}$ Kulliyyah of Pharmacy, International Islamic University Malaysia, 25200 Kuantan, Pahang, Malaysia. ${ }^{4}$ Tobacco Control Center-WHO Collaborating Center, Hamad Medical Corporation, Doha, Qatar. ${ }^{5}$ Weil Cornell Medicine-Qatar, PO Box 24144, Doha, Qatar.

Received: 22 June 2018 Accepted: 21 November 2018 Published online: 08 January 2019

\section{References}

1. The World Health Organization: Tobacco. 2018. http://www.who.int/newsroom/fact-sheets/detail/tobacco. Accessed 12 May 2018.

2. Centers for Disease Control and Prevention: Smoking and Tobacco Use. 2018. https://www.cdc.gov/tobacco/data_statistics/fact_sheets/fast_facts/ index.htm. Accessed 12 May 2018.

3. The World Health Organization: Tobacco Free Initiative fact sheet about health benefits of smoking cessation. 2018. http://www.who.int/tobacco/ quitting/benefits/en/. Accessed 12 May 2018.

4. Centers for Disease Control and Prevention: Quitting Smoking. 2017. https:// www.cdc.gov/tobacco/data_statistics/fact_sheets/cessation/quitting/index. htm. Accessed 12 May 2018.

5. U.S. Department of Health and Human Services: Treating tobacco use and dependence: 2008 update. 2008. https://www.ahrq.gov/professionals/ clinicians-providers/guidelines-recommendations/tobacco/index.html. Accessed 12 May 2018.

6. Anderson C, Blenkinsopp A, Armstrong M. The contribution of pharmacy to improving the public's health. Report 1, Evidence from the peer-reviewed literature. Pharmacy Health Link and Royal Pharmaceutical Society of Great Britain, 2003. ISBN 0-9538505-1-X. http://eprints.nottingham.ac.uk/1571/1/ The_contribution_of_community_pharmacy_to_improving_the_ public\%27s_health_evidence_base_report_1.pdf. Accessed 12 May 2018.

7. Blenkinsopp A, Anderson C, Armstrong M. Systematic review of the effectiveness of community pharmacy-based interventions to reduce risk behaviours and risk factors for coronary heart disease. J Public Health Med. 2003;25(2):144-53.

8. Cipole RJ, Strand LM, Morley PC. Pharmaceutical care practice: The patient-centered approach to medication management. USA: McGrawHill Medical, 2012

9. Saba M, Diep J, Saini B, Dhippayom T. Meta-analysis of the effectiveness of smoking cessation interventions in community pharmacy. J Clin Pharm Ther. 2014;39:240-7.

10. Afzal Z, Pogge E, Boomershine V. Evaluation of a pharmacist and nurse practitioner smoking cessation program. J Pharm Pract. 2017;30(4):406-11.

11. Augustine JM, Taylor AM, Pelger M, Schiefer D, Warholak TL. Smoking quit rates among patients receiving pharmacist-provided pharmacotherapy and telephonic smoking cessation counseling. J Am Pharm Assoc (2003). 2016; 56(2):129-36.

12. Chen T, Kazerooni R, Vannort EM, Nguyen K, Nguyen S, Harris J, et al. Comparison of an intensive pharmacist-managed telephone clinic with standard of care for tobacco cessation in a veteran population. Health Promot Pract. 2014;15(4):512-20.

13. El Hajj MS, Kheir N, Al Mulla AM, Shami R, Fanous N, Mahfoud ZR. Effectiveness of a pharmacist-delivered smoking cessation program in the State of Qatar: a randomized controlled trial. BMC Public Health. 2017;17(1): 215. https://doi.org/10.1186/s12889-017-4103-4.
14. Gong J, Baker CL, Zou KH, Bruno M, Jumadilova Z, Lawrence D, et al. A pragmatic randomized trial comparing telephone-based enhanced pharmacy care and usual care to support smoking cessation. J Manag Care Spec Pharm. 2016;22(12):1417-25.

15. Shen X, Bachyrycz A, Anderson JR, Tinker D, Raisch DW. Improving the effectiveness of pharmacist-assisted tobacco cessation: a study of participant- and pharmacy-specific differences in quit rates. Ann Pharmacother. 2015;49(3):303-10.

16. The International Pharmaceutical Federation. FIP Statement of Policy: the Role of the Pharmacist in Promoting a Tobacco Free Future. 2003. https:// www.fip.org/files/fip/news/tobacco-final2.pdf. Accessed 12 May 2018.

17. Hudmon KS, Corelli RL. ASHP therapeutic position statement on the cessation of tobacco use. Am J Health Syst Pharm. 2009;66:291-307.

18. Adult Tobacco Survey Qatar. 2013. https://www.moph.gov.qa/ar/ publications/Documents/4Link/GATS\%20full\%20report.pdf. Accessed 12 May 2018.

19. The World Health Organization: Global Youth Tobacco Survey Qatar. 2014. https://www.moph.gov.qa/ar/publications/Documents/4Link/GYTS_FS_QAT_ 2013.pdf. Accessed 12 May 2018.

20. Qatar Ministry of Public Health: Current Status and Objectives:

Cardiovascular Diseases. 2018. https://phs.moph.gov.qa/data/cardiovasculardiseases/. Accessed 12 May 2018.

21. Qatar Cancer Country Profile 2014. http://who.int/cancer/country-profiles/ qat en.pdf?ua=1. Accessed 12 May 2018.

22. Smoking-Addressing a National Epidemic. https://qatar-weill.cornell.edu/ Portals/0/Health\%20and\%20Wellness/Documents/SmokingEpidemic.pdf .Accessed 12 May 2018.

23. Law No. 10 of 2016 on the Control of Tobacco and its Derivatives. https:// www.hamad.qa/EN/Hospitals-and-services/QMI/TCC/Documents/TobaccoLaw-EN-AR.pdf. Accessed 12 May 2018.

24. Qatar Ministry of Public Health: Current Status and Objectives: Tobacco cessation. https://phs.moph.gov.qa/data/smoking/. Accessed 12 May 2018.

25. Qatar Council for Healthcare Practitioners: http://www.qchp.org.qa/en/ pages/searchpractitionerspage.aspx. Accessed 9 June 2018.

26. El Hajj MS, Al Nakeeb RR, Al-Qudah RA. Smoking cessation counseling in Qatar: community pharmacists' attitudes, role perceptions and practices. Int J Clin Pharm. 2012;34:667-76.

27. Ye L, Goldie C, Sharma T, John S, Bamford M, Smith PM, Selby P, Schultz ASH. Tobacco-nicotine education and training for health-care professional students and practitioners: a systematic review. Nicotine Tob Res. 2018;20(5):531-42.

28. Association for the Treatment of Tobacco use and Dependence: Core Competencies For Evidence-based Treatment of Tobacco Dependence. 2005. https://www.attud.org/pdf/Standards.pdf. Accessed 12 May 2018.

29. Xu T, de Almeida Neto AC, Moles RJ. A systematic review of simulated patient methods used in community pharmacy to assess the provision of non-prescription medicines. Int J Pharm Pract. 2012;20:307-19.

30. Mesquita AR, de Oliveira Sa DA, Santos AP, de Almeida Neto A, Lyra DP Jr. Assessment of pharmacist's recommendation of non-prescription medicines in Brazil: a simulated patient study. Int J Clin Pharm. 2013;35(4):647-55.

31. Saba M, Diep J, Bittoun R, Saini B. Provision of smoking cessation services in Australian community pharmacies: a simulated patient study. Int J Clin Pharm. 2014;36:604-14.

32. Chiang PP, Chapman S. Do pharmacy staff recommend evidenced-based smoking cessation products? A pseudo patron study. J Clin Pharm Ther. 2006;31(3):205-9.

33. Qatar Ministry of Public Health: Clinical guidelines for the State of Qatar. Management of tobacco dependency. 2016. https://www.moph.gov.qa/ health-strategies/Documents/Guidelines/Tobacco\%20dependency.pdf. Accessed 12 May 2018.

34. Schulz KF, Altman DG, Moher D, for the CONSORT Group. CONSORT 2010 Statement: updated guidelines for reporting parallel group randomized trials. BMJ. 2010;340:c332.

35. Greenhalgh T, Macfarlane F, Steed L, Walton R. What works for whom in pharmacist-led smoking cessation support: realist review. BMC Med. 2016; 14:209.

36. The World Health Organization Europe: WHO European Strategy for Smoking Cessation Policy. 2004. http://www.euro.who.int/_data/assets/ pdf_file/0017/68111/E80056.pdf. Accessed 12 May 2018. 